\title{
Effective Calibration and Evaluation of Multi-Camera Robotic Head
}

\author{
Petra Kocmanova \\ LTR s.r.o. \\ Brno, Czech Republic
}

\author{
Ludek Zalud \\ LTR s.r.o. \\ Brno, Czech Republic
}

\begin{abstract}
The paper deals with appropriate calibration of multispectral vision systems and evaluation of the calibration and data-fusion quality in real-world indoor and outdoor conditions. Checkerboard calibration pattern developed by our team for multispectral calibration of intrinsic and extrinsic parameters is described in detail. The circular object for multispectral fusion evaluation is described as well. The objects were used by our team for calibration and evaluation of advanced visual system of Orpheus-X3 robot that is taken as a demonstrator, but their use is much wider, and authors suggest to use them as testbed for visual measurement systems of mobile robots. To make the calibration easy and straightforward, the authors developed MultiSensCalib program in Matlab, containing all the described techniques. The software is provided as publicly available, including source code and testing images.
\end{abstract}

Keywords-calibration; camera; mobile robot; thermal imaging; data-fusion

\section{INTRODUCTION}

Reconnaissance mobile robotics gains importance during the last years. Visual and space measurement subsystem is typically the most important sensory equipment with most significant impact on mission success. There are many missions in today's society that may require expendable robots to perform exploration in inaccessible or dangerous environments instead of indispensable people. As examples we can name CBRNE (Chemical, biological, radio-logical, nuclear, explosive), counter-terrorist fight, US\&R (Urban Search and Rescue), etc.

Since the missions take place in real world, the robots have to be equipped to most, if not all, possible conditions that may happen. During both military and non-military search and rescue missions, the robot can meet such conditions like complete darkness, smoke, fog, rain, etc. For these conditions, the visual spectrum of humans is not sufficient to provide valuable data. One of the most promising approaches for a wide spectrum of situations is a combination of data from the visual spectrum, near infrared spectrum and far infrared spectrum. In visual spectrum (using standard tricolor cameras), the operator has the best overview of the situation since he/she gets a signal that is most similar to what he knows. By using thermal imagers working in far infrared spectrum he/she can perfectly perceive even slight changes in temperatures. This spectrum very well penetrates through water particles (fog, rain) plus it is not affected by visible light conditions. Most TOF (time-of-flight) proximity scanners and cameras work in the near-infrared spectrum.
The main aim of this paper is the determination of effective sensory head calibration, containing typical sensors for the mentioned situations - tricolor cameras working in the visual spectrum, thermal imagers working in far infrared (FIR) and proximity camera working in near infrared (NIR).

Optimal image configuration is an important factor for effective calibration, so great attention was paid to it.

Calibration of the sensory head was proposed according to Zhang algorithm [1]. Zhang investigated the performance of his one camera calibration algorithm with respect to a number of images of the model plane. A number of images varied from two to 16. The error of intrinsic and extrinsic parameters decreased significantly between calibration from two and three images. Precision improves for more than images only insignificantly.

Calibration performance with respect to the orientation of the model plane was also investigated in [1]. Best performance was achieved with angle $45^{\circ}$ between calibration plane and the image plane. This angle value is difficult to apply in real condition because it decreased the precision of corners extraction.

Photogrammetric software Photomodeler [2] recommends for one camera calibration using minimal six and optimal eight images of calibration plate from different angles. Another recommendation is using less than 12 images for camera lenses with wide angle and high distortion. Next recommendation is making at least two images with a roll of $90^{\circ}$ (camera portrait, landscape orientation). Unfortunately this rotation isn't possible with proposed sensory head, because of the sensory head manipulator.

Bouquet in Complete Camera Calibration Toolbox for Matlab [3] recommends using about 20 images of a planar checkerboard. 6-10 images should be enough for calibration in Omnidirectional Camera Calibration Toolbox for Matlab [4].

Effective image configuration for camera calibration of the sensor head of robot Orheus-X3 will be investigated in this paper.

The organization of the paper is as follows. In Chapter II the used hardware is described. Chapter III deals with calibration process of the camera head. In Chapter IV the datafusion is described, and Chapter $\mathrm{V}$ aims to optimal image configuration and describes evaluation experiments made to evaluate the system. 


\section{HARDWARE}

Although the CASSANDRA robotic system is rather complex and contains several interesting robots, only the Orpheus-X3 is important for the purposes of this paper. CASSANDRA robots are described in detail in [10], [11], [12].

\section{A. Orpheus-X3}

The Orpheus-X3 is an experimental reconnaissance robot based on the Orpheus-AC2 model made by our team to facilitate the measurement of chemical and biological contamination or radioactivity for military. The Orpheus-X3 offers the same drive configuration as its predecessor, namely the four extremely precise AC motors with harmonic gears directly mechanically coupled to the wheels; this configuration makes the robot very effective in hard terrain and enables it to achieve the maximum speed of $15 \mathrm{~km} / \mathrm{h}$. The main difference lies in the chassis, which is not designed as completely waterproof but consists of a series of aluminum plates mounted on a steel frame of welded L-profiles. This modular structural concept makes the robot markedly more versatile, and this is a very important aspect in a robot made primarily for research activities. Furthermore, the device is equipped with a 3DOF manipulator for the sensor head. The manipulator, again, comprises very powerful AC motors combined with extremely precise, low backlash harmonic drive gearboxes made by the Spinea company. The presence of such precise gearboxes can be substantiated by several reasons, mainly by the fact that the robot is used not only for telepresence but also for mobile mapping and SLAM. As currently planned, the robot's only proximity sensor is the TOF camera placed on the sensory head. The Orpheus robots are described in more details in our previous papers, such as [5].

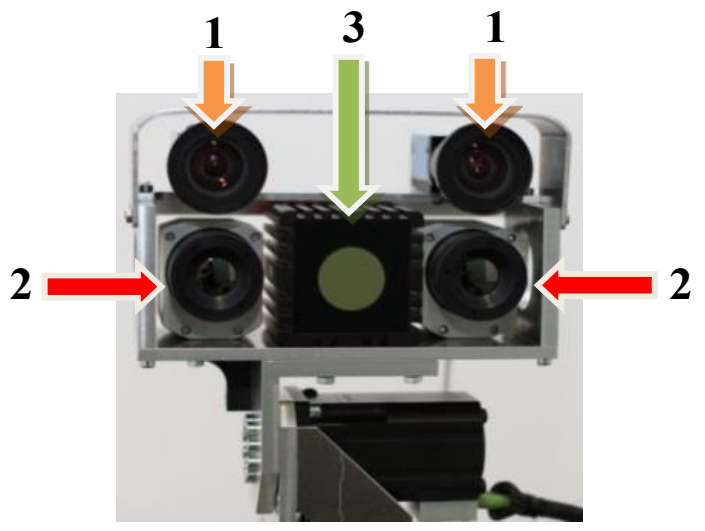

Fig. 1. The sensor head. 1 - tricolor CCD cameras; 2 - thermal imagers; 3 TOF camera

\section{B. Sensor Head}

The sensor head containing five optical sensing elements is shown in Fig. 1. The sensors are as follows:

- Two identical tricolor CCD cameras (see 1 in Fig. 1): TheImagingSource DFK23G445 with the resolution of $1280 \times 960$ pixels, max. refresh rate of $30 \mathrm{~Hz}$, and $\mathrm{GiGe}$ Ethernet protocol. This device is equipped with a Computar $5 \mathrm{~mm} \mathrm{1:1.4} \mathrm{lens.} \mathrm{The} \mathrm{field} \mathrm{of} \mathrm{view} \mathrm{is} 40^{\circ}(\mathrm{h}) \mathrm{x}$ $51^{\circ}(\mathrm{v})$
- Two identical thermal imagers (see 2 in Fig. 1): Flir Tau 640 with the resolution $640 \times 512$, temperature resolution $0.05 \mathrm{~K}$ and Ethernet output. The field of view is $56^{\circ}(\mathrm{h}) \mathrm{x}$ $69^{\circ}(\mathrm{v})$.

- One TOF camera (see 3 in Fig. 1): A Mesa Imaging SR4000 with the range of $10 \mathrm{~m}$, resolution of $176 \times 144$ pixels, and an Ethernet output. The field of view is $56^{\circ}(\mathrm{h}) \times 69^{\circ}(\mathrm{v})$.

The largest FOV capture thermal imagers and the TOF camera, which is required for the simultaneous use of stereovision and thermal stereovision. The main disadvantage of the applied TOF camera is its low number of pixels. Compared to the CCD cameras, it is about 10 times lower in one axis, and in relation to thermal imagers it is 4 times lower.

\section{SENSOR HEAD CALIBRATION}

Here will be described only calibration of intrinsic and extrinsic parameters. It is also necessary to calibrate temperatures of thermal imagers, in detail described in [6] and TOF camera measured distances, this calibration is described in detail in [7].

First condition for successful calibration is calibration plate with pattern visible in all 3 used spectrums:

- Infrared for TOF camera $(850 \mathrm{~nm})$.

- Visible spectrum for CCD cameras.

- Long-wavelength infrared for thermal imagers.

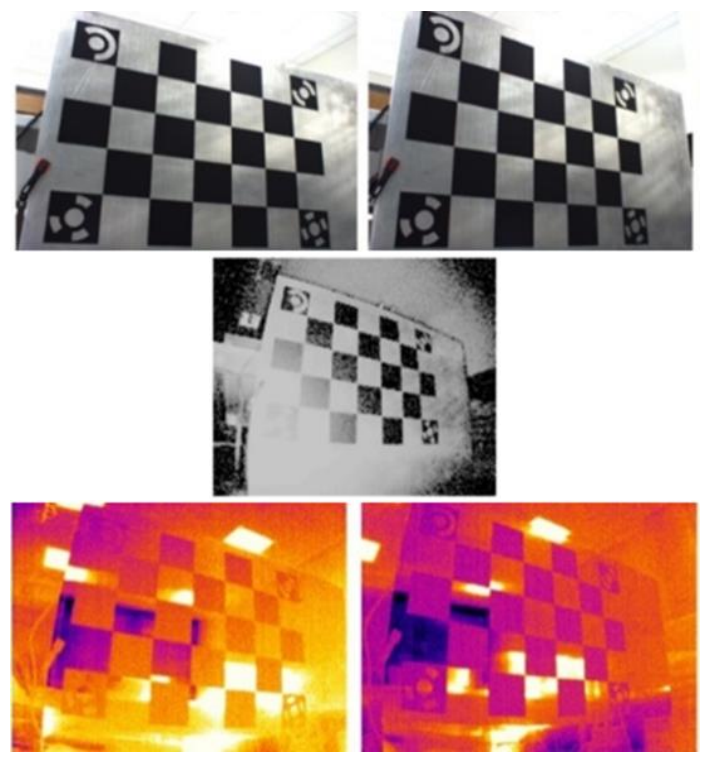

Fig. 2. The initial calibration plate: the left and right CCD cameras (up); the TOF camera intensity image (center). the left and right thermal imager

Three calibration plate based on checkerboard pattern were proposed. At first sufficient contrast of the calibration pattern should be achieved only by different materials. This version comprised an aluminum panel (low emissivity; high reflectivity) and a self-adhesive foil (high emissivity; low reflectivity). The main problem related to this initial board consisted in the high reflectivity of the aluminum base in cases 
that images are acquire under non-perpendicular angle (see Fig. 2).

The second version consisted of an aluminum panel with a laser-cut, anodized pattern and chipboard covered by a black, matt foil. Anodizing of aluminum panel reduces high reflectivity. Good contrast of checkerboard pattern for thermal imagers was achieved by heating of aluminum part at $50^{\circ} \mathrm{C}$.

The final version included a $2 \mathrm{~mm}$ laser-cut aluminum plate with active heating. This version is more comfortable and shortens time needed to prepare calibration (see Fig. 3).

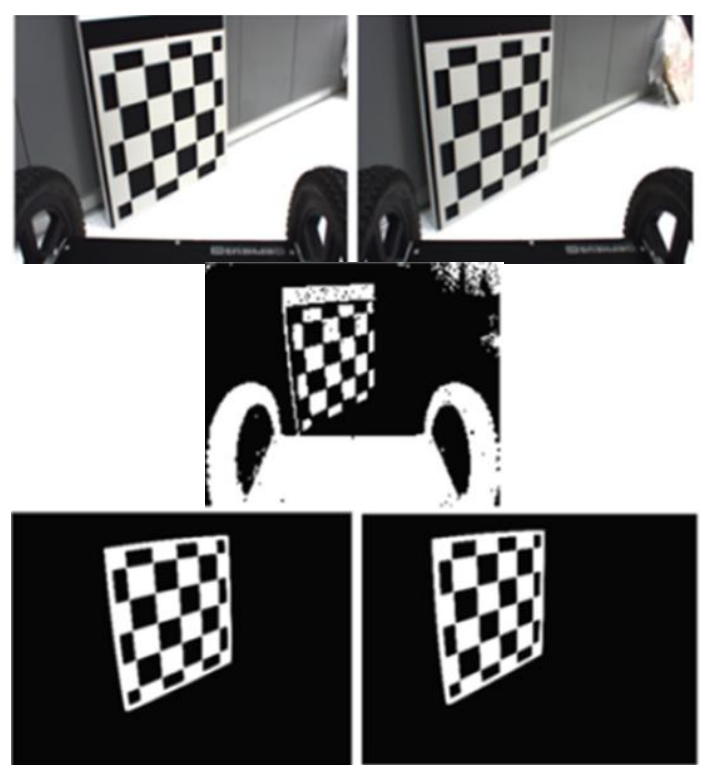

Fig. 3. The final calibration plate: the left and right CCD cameras (up); the TOF camera intensity image (center). the left and right thermal imager cameras (down)

Software MultiSensCalib was created for calibration of intrinsic and extrinsic parameters of sensor head camera system. The calibration comprises the following stages:

- Corner extraction based on automatic corner extraction from Omnidirectional Camera Calibration Toolbox for Matlab [4].

- Homography from extracted corners.

- Intrinsic and extrinsic parameters are computed from homography according to [1].

- Nonlinear optimization that minimizes the sum of the squares of the re-projection errors including the determination of distortion first for each camera separately and then for all together.

More details about calibration are described in [7].

The authors decided to make the software, including source code, publicly available. The executable, Matlab source code and sample images are available for download at http://www.ludekzalud.cz/multisenscalib/

\section{DATA FUSION}

Data fusion is performed by means of image transformations. The range measurements of the TOF camera can be displayed into images of the CCD cameras and thermal imagers using spatial coordinates. The thermal image can be displayed into the CCD image according to identical points (ID) of the TOF camera transformed into frames of the CCD camera and the thermal imager and vice versa (see Fig. 4).

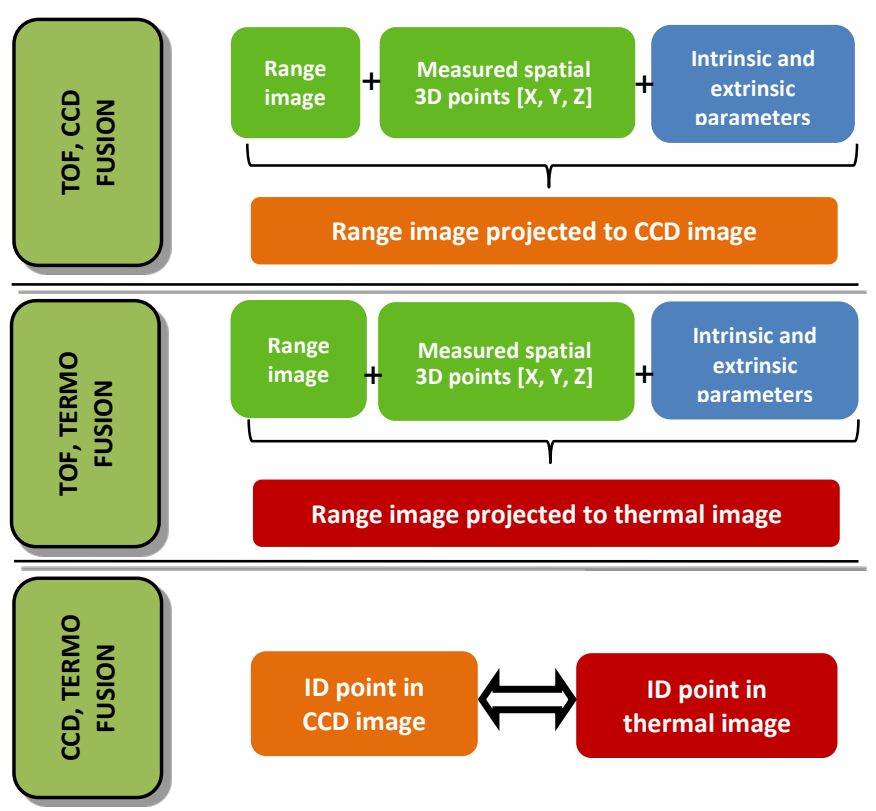

Fig. 4. Scheme of data fusion: TOF and CCD data fusion (up); TOF and thermal data fusion (centre); CCD and thermal data fusion (down)

The input data for data fusion include the range measurement, the image coordinates of all sensors, and the results of previous calibration. The procedure comprises the following stages:

- Computation of spatial coordinates measured by TOF camera.

- Homogeneous transformation to determine measured spatial coordinates in frames of other cameras.

- Perspective projection to determine image coordinates in frames of other cameras.

- Correction of recalculated image coordinates to the calibrated position of the principal point.

The spatial coordinates $X, Y$, and $Z$ are computed according to (1) and (4), where $x, y$ are image coordinates of TOF camera, $f$ focal length and $d_{0}$ is measured distance projected on optical axis. Calculation of spatial coordinate $Z$ in (2) is simplified by substitution of cyclometric function (3).

$$
\begin{array}{r}
X=\frac{d_{0} x}{f}, Y=\frac{d_{0} y}{f} . \\
Z=d_{0}=d \cos \left(\arctan \left(\frac{y}{\sqrt{f^{2}+x^{2}}}\right)\right) \cos \left(\arctan \left(\frac{x}{f}\right)\right)
\end{array}
$$




$$
\begin{aligned}
& \cos \left(\tan ^{-1} a\right)=\frac{1}{\sqrt{1+a^{2}}} \\
& Z=\frac{d f}{\sqrt{x^{2}+y^{2}+f^{2}}}
\end{aligned}
$$

The homogeneous transformation is determined by (5), where $R_{[3 \times 3]}$ is the rotational matrix, $t_{[3 \times 1]}$ is the translation vector, and $X^{\prime}, Y^{\prime}, Z^{\prime}$ are the spatial coordinates of the second sensor. The image coordinates of the TOF camera in the next frame $x_{c}{ }^{\prime}, y_{c}{ }^{\prime}$ are computed using perspective projection (6), where $f^{\prime}$ is the focal length of the second sensor.

$$
\begin{aligned}
& {\left[\begin{array}{c}
X^{\prime} \\
Y^{\prime} \\
Z^{\prime} \\
1
\end{array}\right]=\left[\begin{array}{ll}
R & t \\
0 & 1
\end{array}\right]\left[\begin{array}{c}
X \\
Y \\
Z \\
1
\end{array}\right]} \\
& x_{c}{ }^{\prime}=\frac{f^{\prime} X^{\prime}}{Z^{\prime}}, y_{c}{ }^{\prime}=\frac{f^{\prime} Y^{\prime}}{Z^{\prime}}
\end{aligned}
$$

\section{A. Simulation of the most significant errors}

Errors of sensors on sensor head, for that we expect the most significant impact on data fusion, were simulated. TOF camera is an indispensable element of data fusion, but less accurate than other cameras, therefore were simulated following 2 errors:

- Influence of TOF camera distance error on data fusion precision.

- Influence of low resolution of TOF camera.

The first will be discussed influence of TOF camera distance error. We determined pixel differences caused by TOF camera radial distance error for both CCD cameras and thermal imagers. We simulated distance error for 2 significant image points:

- Point on optical axis of the TOF camera.

- Point on the edge of the region 3 lying on the $\mathrm{x}$-axis. Definition of TOF camera regions is determined in [8].

Measured distances in the region 4 have very low reliability, therefore this region isn't considered. The range of the radial distance simulation is the same as detection range of TOF camera i.e. $0.1-10.0 \mathrm{~m}$.

The effect of distance error is not significant for data fusion if transformed image coordinate differences (CCD cameras and thermal imagers) not exceed 0.5 pixel. For simulation we used values based on distance error from range calibration [7]. It is also important to judge the usefulness and impact of the range calibration. Distance error before calibration $63 \mathrm{~mm}$ and after calibration $30 \mathrm{~mm}$ was used for point on optical axis. Analogously $95 \mathrm{~mm}$ and $50 \mathrm{~mm}$ for point on the edge of reg. 3 .

Fig. 5, Fig. 6, and TABLE I. show effect of pixel error in transformed images caused by distance error. Thin lines denote simulated pixel differences before TOF camera distance calibration, bold lines after. Graphs for point on the edge of reg. 3 have the same character as Fig. 5 and
Fig. 6. The numerical difference is apparent from Table I.. For point on the edge of reg. 3 are pixel error slightly higher than point on optical axis (reg. 1).

Distance error is insignificant for radial distance greater than approximately $2.8 \mathrm{~m}$ for $\mathrm{CCD}$ cameras after range calibration and approximately $1.7 \mathrm{~m}$ in $\mathrm{x}$ axis and $0.3 \mathrm{~m}$ in $\mathrm{y}$ axis for thermal imagers after range calibration. Low influence of distance error on coordinate $y$ of thermal imagers is caused by mounting of TOF camera and thermal imagers in the same height level on sensor head. Table I. also shows contribution of TOF camera distance calibration for more precise data fusion especially for objects in lower distances.

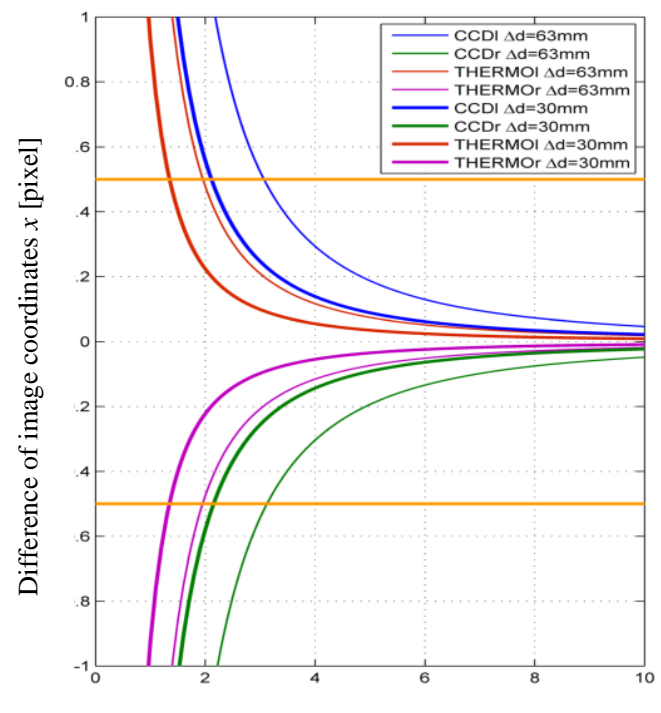

Radial distance along TOF camera optical axis [m]

Fig. 5. Image coordinate differences $\Delta x$ caused by distance error for point on optical axis of TOF camera

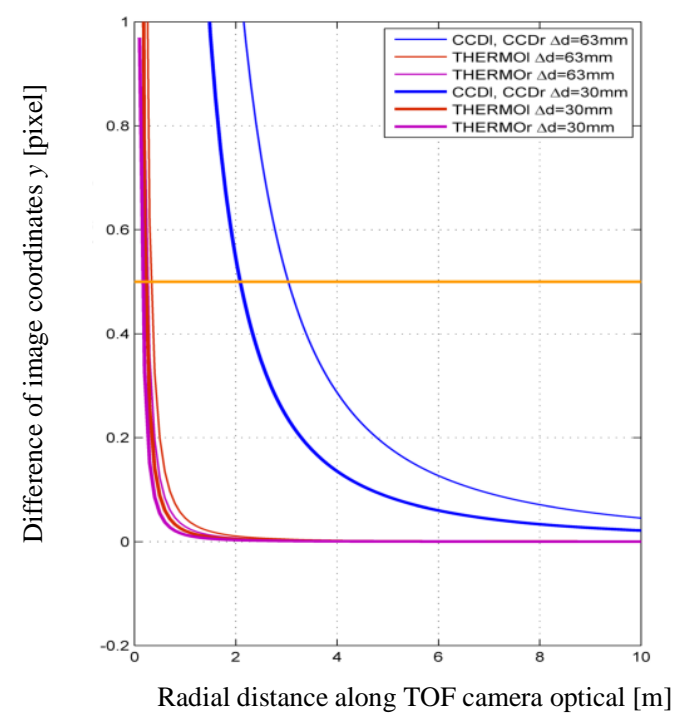

Fig. 6. Image coordinate differences $\Delta y$ caused by distance error for point on optical axis of TOF camera 
TABLE I. IMAge COORDINATE DifFERENCES 0.5 PiXel CAUSED By TOF CAMERA DisTANCE ERROR

\begin{tabular}{|c|c|c|c|c|c|c|}
\hline & \multirow[t]{2}{*}{$\begin{array}{l}\text { Distance } \\
\text { error }\end{array}$} & \multirow[t]{2}{*}{$\begin{array}{l}\text { Image } \\
\text { coord. }\end{array}$} & \multicolumn{4}{|c|}{$\begin{array}{l}\text { Radial distance at that pixel error } \\
\text { causes by distance error is } 0.5 \text { pixel } \\
\text { [m] }\end{array}$} \\
\hline & & & $C C D l$ & $C C D r$ & TH. $l$ & TH. $r$ \\
\hline \multirow{4}{*}{$\begin{array}{l}\text { Point } \\
\text { on } \\
\text { optical } \\
\text { axis }\end{array}$} & \multirow{2}{*}{$\begin{array}{l}63 \mathrm{~mm} \\
\text { before } \\
\text { calibration }\end{array}$} & $x$ & 3,08 & 3,14 & 1,95 & 1,95 \\
\hline & & $y$ & 3,05 & 3,05 & 0,34 & 0,27 \\
\hline & \multirow{2}{*}{$\begin{array}{l}30 \mathrm{~mm} \\
\text { after } \\
\text { calibration }\end{array}$} & $x$ & 2,12 & 2,15 & 1,36 & 1,35 \\
\hline & & $y$ & 2,09 & 2,09 & 0,23 & 0,17 \\
\hline \multirow{4}{*}{$\begin{array}{l}\text { Point } \\
\text { on the } \\
\text { edge } \\
\text { of } \\
\text { reg. } 3\end{array}$} & \multirow{2}{*}{$\begin{array}{l}95 \mathrm{~mm} \\
\text { before } \\
\text { calibration }\end{array}$} & $x$ & 3,78 & 3,85 & 2,411 & 2,40 \\
\hline & & $y$ & 3,75 & 3,75 & 0,42 & 0,34 \\
\hline & \multirow{2}{*}{$\begin{array}{l}50 \mathrm{~mm} \\
\text { before } \\
\text { calibration }\end{array}$} & $x$ & 2,74 & 2,79 & 1,74 & 1,74 \\
\hline & & $y$ & 2,72 & 2,72 & 0,30 & 0,23 \\
\hline
\end{tabular}

Influence of low resolution of TOF camera depending on TOF camera image radial distance is the second investigated problem. Results of this simulation reflect different resolution of cameras as expected. Error 0.5 pixel in the image of TOF camera cause an error of image coordinate $x, y$ for CCD cameras approximately 5 pixel of CCD camera and for thermal imagers approximately 2 pixel of thermal imager (see Fig. 7 and Fig. 8).

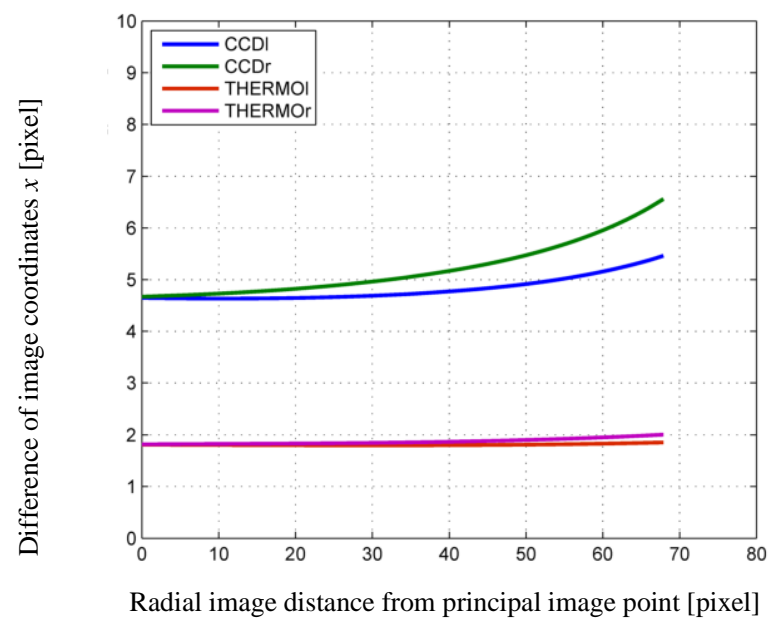

Fig. 7. Image coordinate differences $\Delta \mathrm{x}$ caused by error 0.5 pixel in TOF camera image coordinates

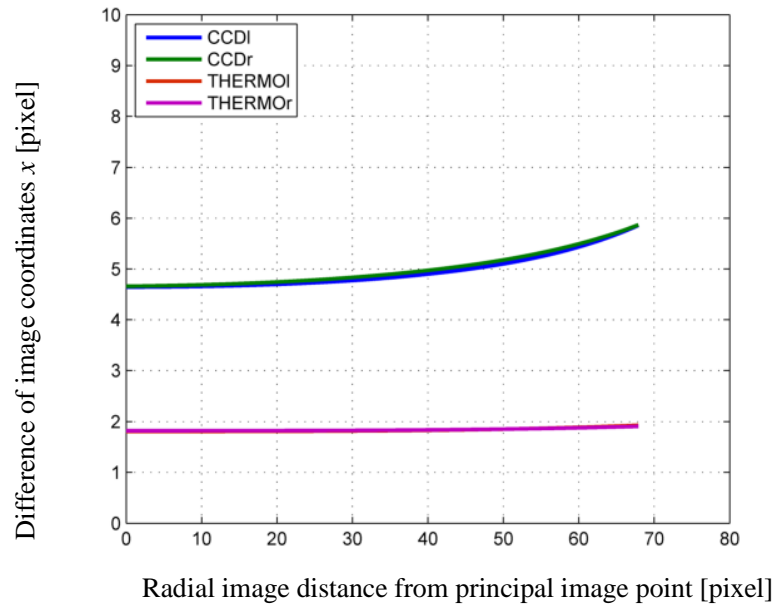

Fig. 8. Image coordinate differences $\Delta \mathrm{x}$ caused by error 0.5 pixel in TOF camera image coordinates

\section{OPtimal IMAGE CONFIGURATION}

Selection of appropriate image configuration is a vital part of the whole calibration process and it has great impact to calibration results, and subsequently to multispectral datafusion quality and robustness. To choose the most appropriate configuration, we started with 10 image configurations, see 0 Blue dots in second column denote image in normal position and blue arrows denote direction of image acquisition. 2-9 images were used for sensory head calibration. Edges of images that do not contain calibration target, are greater than usually, because of cameras rotations in sensory head and different fields of view.

The most effective configuration was determined according to independent evaluation of data fusion precision.

The principle of this evaluation is comparison of identical objects directly extracted from images from CCD cameras and thermal imagers with objects extracted from images from TOF camera and projected to CCD cameras and thermal imagers frames, using data fusion algorithm.

We had to propose objects for this verification that may be easily identifiable in the all corresponding images. 
TABLE II. INVESTIGATED IMAGE CONFIGURATIONS FOR SENSORY HEAD CALIBRATION

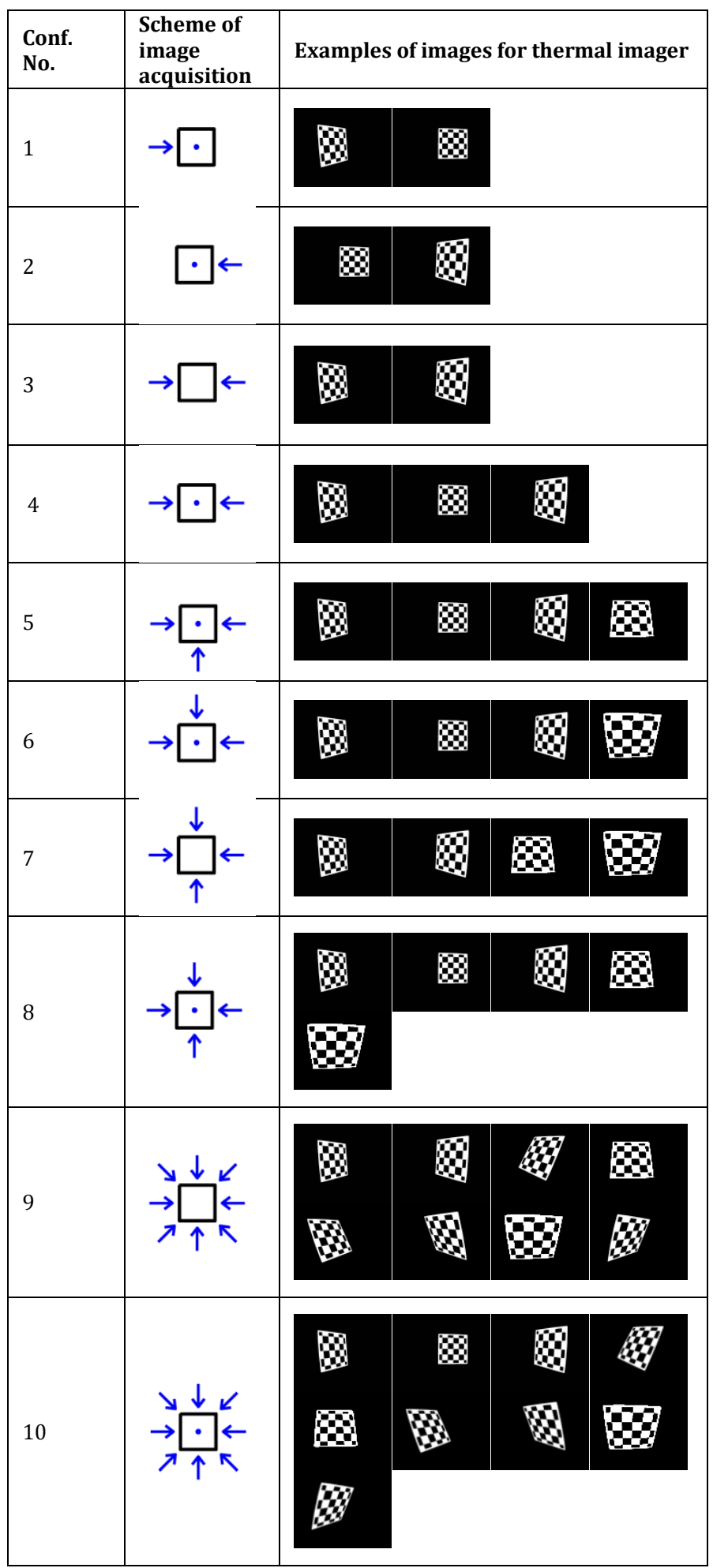

The first our design of this object was sphere. The main reason for such choice arose from the fact that the robot moves around the objects to be identified, and it is vital that they appear identically from all points of view. The spheres can be recognized without difficulty in a color image (Fig. 9 up). In the thermal image, the identification was carried out simply via heating the metal spheres to $60^{\circ} \mathrm{C}$ before the measurement (Fig. 9 down). We used petanque balls $(72 \mathrm{~mm}$ in diameter) and a shot put ball (104 $\mathrm{mm}$ in diameter). The most difficult problem was to recognize the spheres in the TOF camera images. Although spherical objects are commonly used for terrestrial scan registering [9], metal balls could not be reliably identified mainly due to low spatial resolution of the used TOF camera, range errors, noise, and size of the spheres.

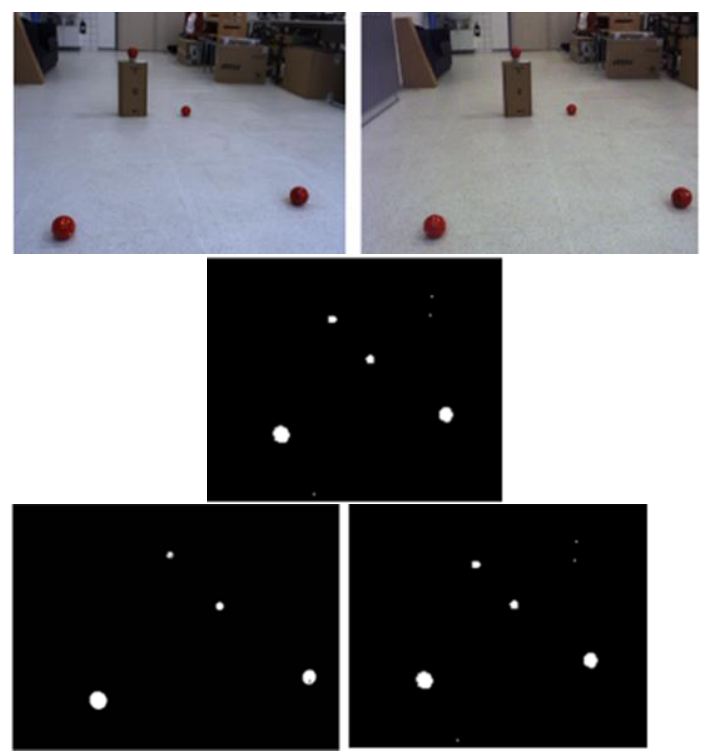

Fig. 9. The images of first target for the verification of the data fusion accuracy: the left and right CCD cameras (up); the TOF camera intensity image (center). the left and right thermal imager cameras (down)

Final design of target clearly identifiable in images of all cameras was aluminum circle covered with black paper in the middle and with $3 \mathrm{M}$ red reflective tape on the edge with active heating. Reflective tape is used for easier identification of targets in images of TOF camera, but significant disadvantages of this reflectivity is missing measured distances, since too big portion of light is returned unidirectional. The matte paper in the middle of the circle was used to overcome this problem - it is easy-to-be-identified by the TOF camera. We used 3 aluminum circles with $20 \mathrm{~cm}$ and $30 \mathrm{~cm}$ diameters. The targets are well identifiable on images of all 3 camera types (see Fig. 10). 


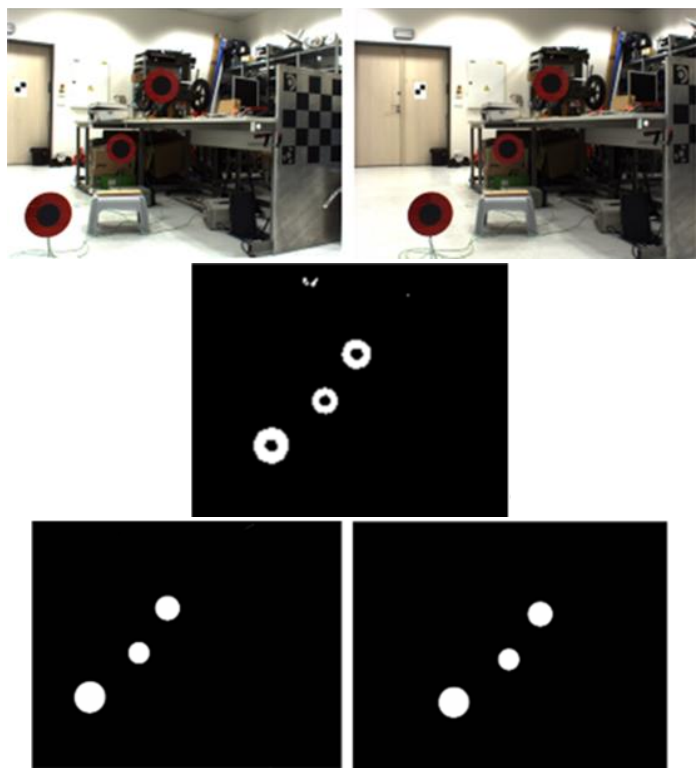

Fig. 10. The images of final target for the verification of the data fusion accuracy: the left and right CCD cameras (up); the TOF camera intensity image (center). the left and right thermal imager cameras (down)

Eighty-seven images were obtained in the experiment under real indoor conditions from the free ride of the robot. 211 extracted objects were used for data fusion evaluation, TOF camera image radial distance for these objects was in range from 1-67 pixels, range for measured distance was from 1.1 to $5.7 \mathrm{~m}$.

Extraction of targets from images comprises the following stages:

- Thresholding.

- Removing small objects (noise) using morphological opening.

- Connection of separated parts using morphological closing.

- Filling closed objects.

- Determining of centroid coordinates.

0shows standard deviations $\sigma_{x}, \sigma_{y}$ of image coordinates $x, y$ projected by proposed data fusion algorithm for tested configurations 4-10. Standard deviation of image coordinates is denoted as $\sigma$. Values of standard deviation are given in pixels of CCD cameras and thermal imagers. Values of intrinsic and extrinsic parameters computed from only 2 images, i.e. configuration 1-3, are far away from real values.

The most suitable configurations according to values of standard deviations are configuration 8 and 10 , but configuration 8 contains error increasing with image radial distance, in detail described in [7]. Configuration 10 of images is reliable according to proposed evaluation of data fusion.
TABLE III. STANDARD DEVIAATIONS OF IMAGE COORDINATES FOR CONFIGURATIONS 4-10

\begin{tabular}{|c|c|c|c|c|c|}
\hline \multirow{2}{*}{ Conf. No } & & \multicolumn{4}{|c|}{ Standard deviation od data fusion [pixel] } \\
\hline & & $C C D l$ & $C C D r$ & TH. l & TH. $r$ \\
\hline \multirow{3}{*}{4} & $\sigma_{x}$ & 6.3 & 6.3 & 2.2 & 3.5 \\
\hline & $\sigma_{y}$ & 5.2 & 6.0 & 2.3 & 2.3 \\
\hline & $\sigma$ & 5.8 & 6.2 & 2.3 & 3.0 \\
\hline \multirow{3}{*}{5} & $\sigma_{x}$ & 2.8 & 3.3 & 1.4 & 1.5 \\
\hline & $\sigma_{y}$ & 3.9 & 3.8 & 1.6 & 1.1 \\
\hline & $\sigma$ & 3.4 & 3.6 & 1.5 & 1.3 \\
\hline \multirow{3}{*}{6} & $\sigma_{x}$ & 2.7 & 3.1 & 1.3 & 1.1 \\
\hline & $\sigma_{y}$ & 3.3 & 3.3 & 1.7 & 1.1 \\
\hline & $\sigma$ & 3.0 & 3.2 & 1.5 & 1.1 \\
\hline \multirow{3}{*}{7} & $\sigma_{x}$ & 3.7 & 3.4 & 1.3 & 1.3 \\
\hline & $\sigma_{y}$ & 3.9 & 3.7 & 1.3 & 1.3 \\
\hline & $\sigma$ & 3.8 & 3.6 & 1.3 & 1.3 \\
\hline \multirow{3}{*}{8} & $\sigma_{x}$ & 2.0 & 2.4 & 1.2 & 1.1 \\
\hline & $\sigma_{y}$ & 3.2 & 3.2 & 1.5 & 1 \\
\hline & $\sigma$ & 2.7 & 2.8 & 1.4 & 1.1 \\
\hline \multirow{3}{*}{9} & $\sigma_{x}$ & 2.9 & 3.5 & 1.2 & 1.1 \\
\hline & $\sigma_{y}$ & 4.1 & 4.4 & 1.4 & 1.2 \\
\hline & $\sigma$ & 3.6 & 4.0 & 1.3 & 1.2 \\
\hline \multirow{3}{*}{10} & $\sigma_{x}$ & 2.4 & 3.0 & 1.0 & 1.2 \\
\hline & $\sigma_{y}$ & 3.2 & 3.3 & 1.2 & 1.1 \\
\hline & $\sigma$ & 2.8 & 3.2 & 1.1 & 1.2 \\
\hline
\end{tabular}

The differences between the extracted centroid coordinates and those projected from TOF image using data fusion algorithm depending on TOF image radial distances is displayed in Fig. 11-Fig. 18for configuration 10. Due to the fact that the TOF camera has the lowest resolution, the following figure shown regions that include errors in TOF image centroid extraction in range $-0.5-+0.5$ pixel (delimited by the orange horizontal lines).

The boundaries of TOF camera distance measurement accuracy regions are displayed in the following figures.

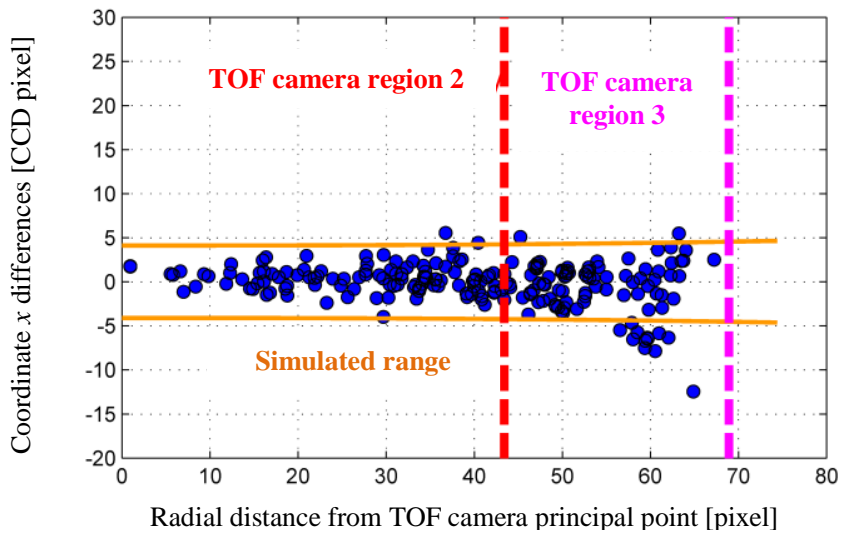

Fig. 11. The coordinate differences determined from the extracted centroids in images of the left CCD camera and from projected TOF image coordinates using the data fusion algorithm: the coordinate $x$ differences 


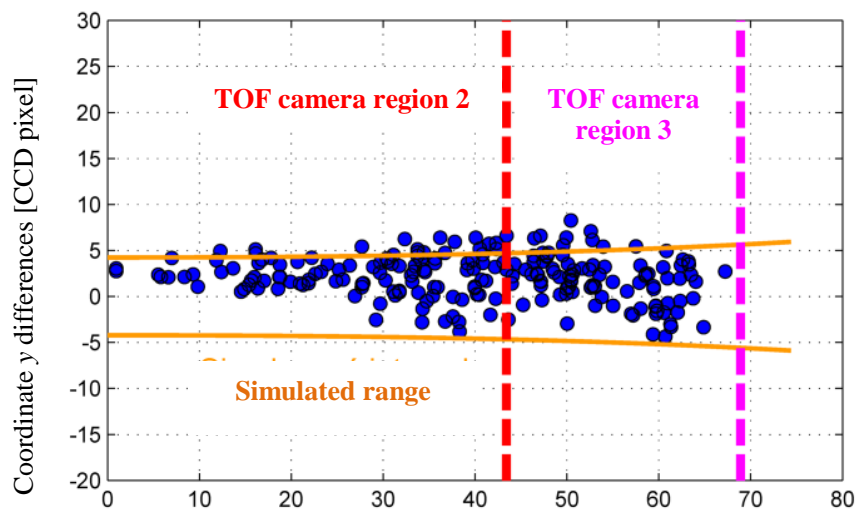

Radial distance from TOF camera principal point [pixel]

Fig. 12. The coordinate differences determined from the extracted centroids in images of the left CCD camera and from projected TOF image coordinates using the data fusion algorithm: the coordinate $y$ differences

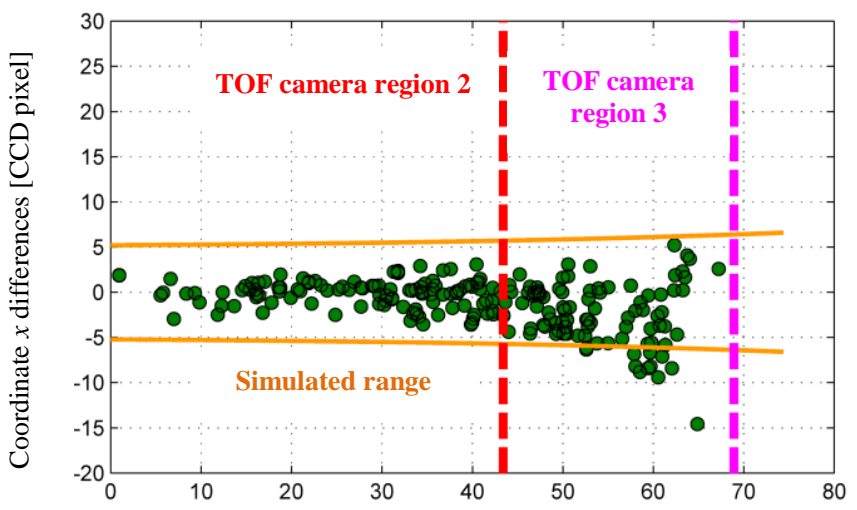

Radial distance from TOF camera principal point [pixel]

Fig. 13. The coordinate differences determined from the extracted centroids in images of the right CCD camera and from projected TOF image coordinates using the data fusion algorithm: the coordinate $x$ differences

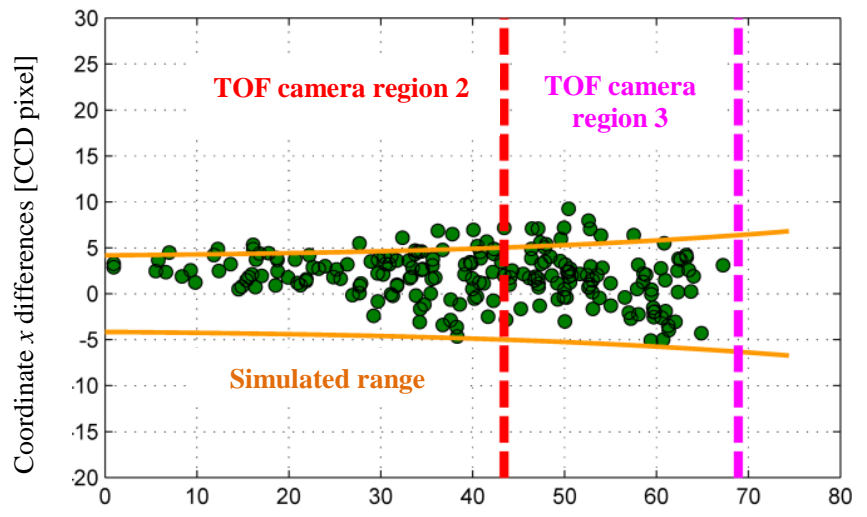

Radial distance from TOF camera principal point [pixel]

Fig. 14. The coordinate differences determined from the extracted centroids in images of the right CCD camera and from projected TOF image coordinates using the data fusion algorithm: the coordinate $y$ differences

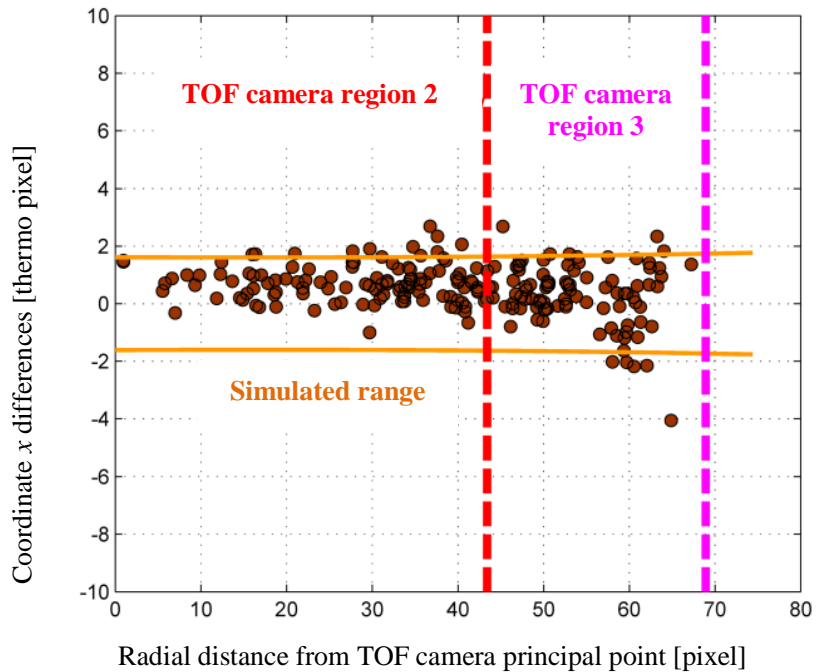

Fig. 15. The coordinate differences determined from the extracted centroids in images of the left thermal imager and from projected TOF image coordinates using the data fusion algorithm: the coordinate $x$ differences

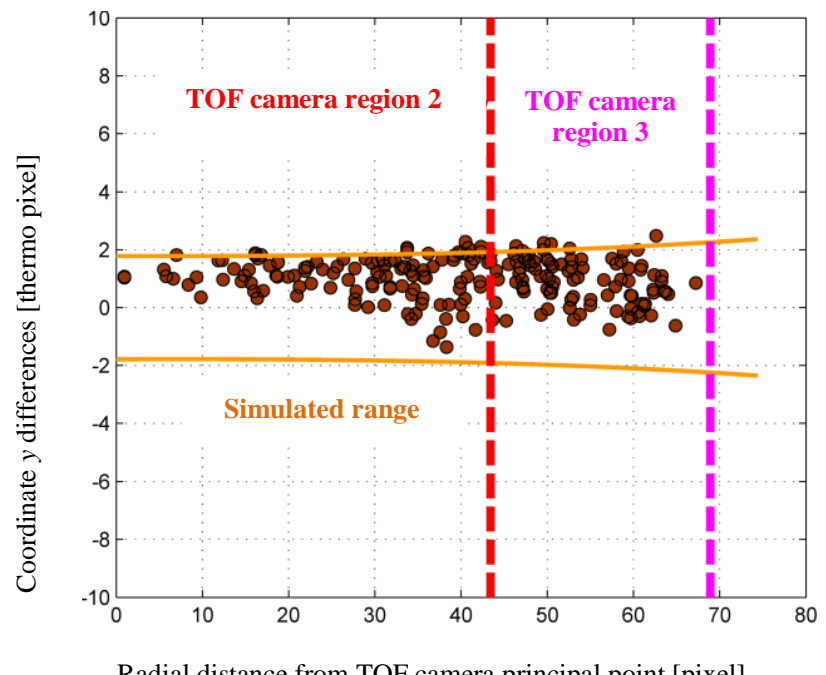

Fig. 16. The coordinate differences determined from the extracted centroids in images of the left thermal imager and from projected TOF image coordinates using the data fusion algorithm: the coordinate $y$ differences 


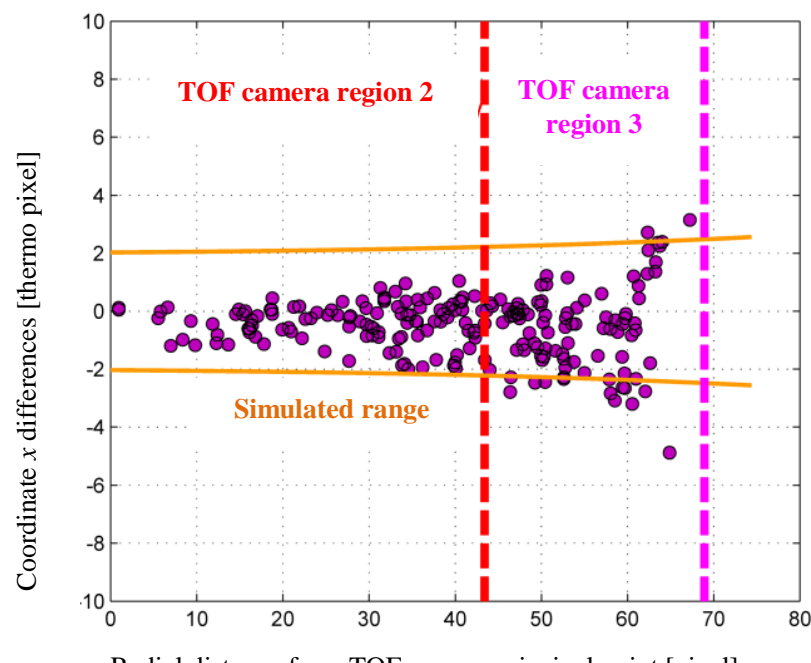

Radial distance from TOF camera principal point [pixel]

Fig. 17. The coordınate ditterences determıned trom the extracted centroids in images of the right thermal imager and from projected TOF image coordinates using the data fusion algorithm: the coordinate $x$ differences

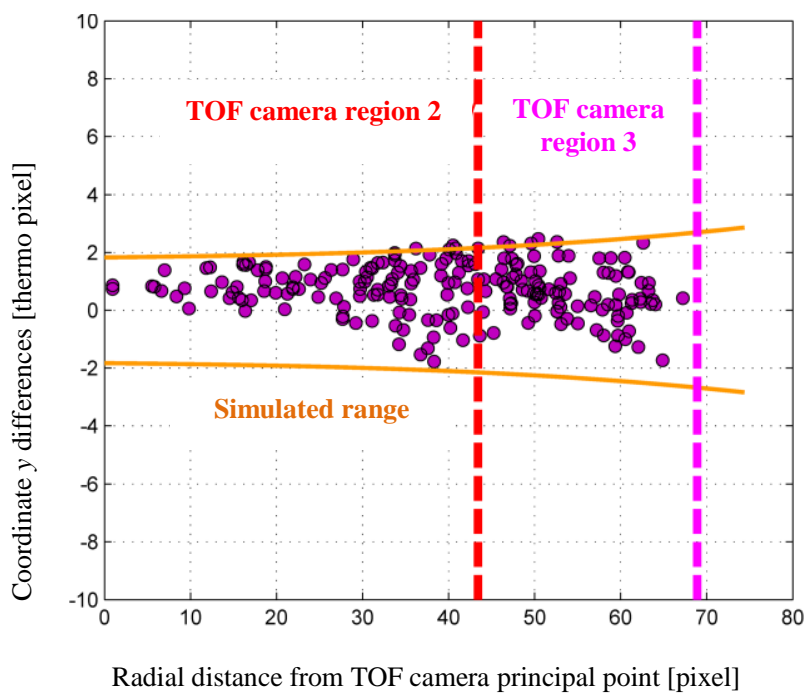

Fig. 18. The coordinate differences determined from the extracted centroids in images of the right thermal imager and from projected TOF image coordinates using the data fusion algorithm: the coordinate $y$ differences

\section{CONCLUSION}

As it is apparent from evaluation experiment described in Chapter 4, the fusion described in Chapter 3 is possible, but has its limits. The main problems come from the fact, the cameras used in the described case have significantly different spatial pixel resolution. It has to be said the cameras were carefully selected to have parameters appropriate for Orpheus-X3 robot's main mission - real-time telepresence with augmented reality containing thermal information. The cameras had to be small, lightweight, but they also offered unusually wide fieldof-view. We can suppose for bigger robots sensors with considerably higher resolution might be used. The sensor resolution will also evolve in time (thermal cameras, 3D proximity cameras).
Numerical evaluation of data fusion algorithm is as follows: standard deviation for $x, y$ image coordinates is around three pixels for CCD cameras (0.3 Pixel of TOF camera) and around 1 pixel for thermal imagers (around 0.5 TOF camera pixels).

The presented calibration process and evaluation may be used for visual and optical measurement systems of mobile robots, in general, so its use is much wider than on presented Orpheus- $\mathrm{X} 3$ robot demonstrator.
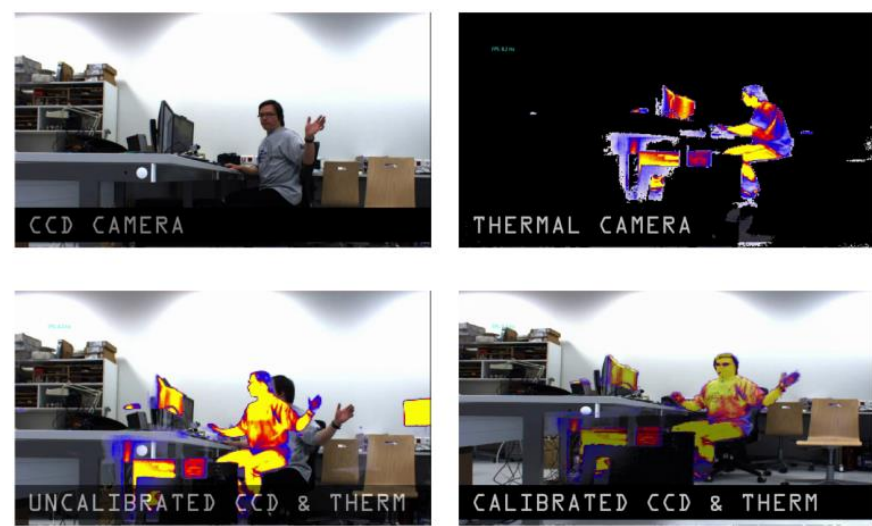

Fig. 19. Image of CCD camera (upper left), image from the thermal imager (upper right), uncalibrated data fusion (bottom left), calibrated data fusion (bottom right)

To make the calibration fast and user-friendly, we developed application MultiSensCalib in Matlab, which is available both in executable and source code in http://www.ludekzalud.cz/multisenscalib/ The same webpage also contains a set of testing images from Orpheus-X3's sensory head and a brief description of the software usage.

For the future, the authors plan to solve the biggest issue of the current data-fusion system - time latency between corresponding images. Currently if the sensory head moves rapidly, the thermal image is delayed after the camera image. Each raw data image will be equipped with time-stamp in the beginning of the data-flow and correspondingly processed. The authors also work on optimization of the algorithms to fasten the necessary processing part.

\section{ACKNOWLEDGMENT}

This work was supported by VG 20122015096 grant named Cooperative Robotic Exploration of Dangerous Areas by Ministry of Interior, Czech Republic, program BV II/2-VS.

This work was supported by the project CEITEC - Central European Institute of Technology (CZ.1.05/1.1.00/02.0068) from the European Regional Development Fund and by the Technology Agency of the Czech Republic under the project TE01020197 "Centre for Applied Cybernetics 3".

\section{REFERENCES}

[1] Z. Zhang, "Flexible camera calibration by viewing a plane from unknown orientations", in Proceedings of the Seventh IEEE International Conference on Computer Vision. 1999. DOI: 10.1109/iccv.1999.791289.

[2] PhotoModeler Pro 5 help. Eos System Inc.: 210 - 1847 West Broadway, Vancouver BC V6J 1Y6, Canada. 
[3] J.-Y. Bouguet,"Complete Camera Calibration Toolbox for Matlab" [online] http://www.vision.caltech.edu/bouguetj/calib_doc/

[4] D. Scaramuzza,"OCamCalib: Omnidirectional Camera Calibration Toolbox for Matlab" [online]. https://sites.google.com/site/scarabotix/ocamcalib-toolbox

[5] L. Zalud, F. Burian, L. Kopecny, and P. Kocmanova," Remote Robotic Exploration of Contaminated and Dangerous Areas", in International Conference on Military Technologies, pp 525-532, Brno, Czech Republic, ISBN 978-80-7231-917-6, 2013

[6] L. Zalud and P. Kocmanova, "Fusion of thermal imaging and CCD camera-based data for stereovision visual telepresence", in 2013 IEEE International Symposium on Safety, Security, and Rescue Robotics (SSRR). Linkoping: IEEE, 2013, pp. 1-6. DOI: 10.1109/SSRR.2013.6719344. ISBN 978-1-4799-0880-6

[7] Zalud L., Kocmanova P., Burian F. et al., "Calibration and Evaluation of Parameters in A 3D Proximity Rotating Scanner", ELEKTRONIKA IR ELEKTROTECHNIKA, Volume 21, Issue 1, pp 3-12, DOI: 10.5755/j01.eee.21.1.7299, Kuanas univ. technol., Lithuania, ISSN $1392-1215,2015$
[8] SR4000 Data Sheet, MESA Imaging AG. Rev. 5.1, 2011.

[9] El khrachy Ismail Abd El hamid Mohamed, "Towards an automatic registration for terrestrial laser scanner data. Braunschweig". 2007. Ph.D. thesis. Technische Universität Carolo-Wilhelmina.

[10] Zalud L., Burian F., Kopecny L., Kocmanova P., "Remote Robotic Exploration of Contaminated and Dangerous Areas", International Conference on Military Technologies, pp 525-532, Brno, Czech Republic, ISBN 978-80-7231-917-6. (2013)

[11] Zalud L., Kopecny L., Burian F., "Robotic Systems for Special Reconnaissance“, ICMT'09: INTERNATIONAL CONFERENCE ON MILITARY TECHNOLOGIES, pp 531-540, Brno, Czech Republic, ISBN 978-80-7231-649-6, 2009

[12] Nejdl L., Kudr J., Cihlarova K. et al, "Remote-controlled robotic platform ORPHEUS as a new tool for detection of bacteria in the environment", Electrophorensis, Volume 35, Issue 16, pp 2333-2345, Wilwy-Blackwell, USA, DOI 10.1002/elps.201300576, ISSN 01730835, 2014 